\title{
Coordinated Tracking for Nonlinear Multiagent Systems under Variable-Time Impulsive Control
}

\author{
Yuan Tian $\mathbb{D}^{1},{ }^{1,2}$ Chuandong Li, ${ }^{1}$ Xujun Yang, ${ }^{1}$ and Yiyan Han ${ }^{1}$ \\ ${ }^{1}$ National \& Local Joint Engineering Laboratory of Intelligent Transmission and Control Technology (Chongqing), \\ College of Electronic and Information Engineering, Southwest University, Chongqing 400715, China \\ ${ }^{2}$ Faculty of Built Environment, Chongqing University, Chongqing 400044, China \\ Correspondence should be addressed to Yuan Tian; tianyuan@cqu.edu.cn
}

Received 28 January 2019; Accepted 16 April 2019; Published 20 May 2019

Academic Editor: Carlos F. Aguilar-Ibáñez

Copyright (C) 2019 Yuan Tian et al. This is an open access article distributed under the Creative Commons Attribution License, which permits unrestricted use, distribution, and reproduction in any medium, provided the original work is properly cited.

\begin{abstract}
This paper addresses variable-time impulsive control for coordinated tracking problem in nonlinear multiagent systems. To make followers coordinately track the leader, a variable-time impulsive controller is designed. Under some well-selected conditions, the comparison system of variable-time impulsive tracking control system is constructed by employing B-equivalence method. And we theoretically demonstrate that the two systems have the same stability property. Coordinated tracking criteria of multiagent systems are obtained by considering the comparison system. Numerical simulation is also provided to illustrate the correctness of theoretical results and the efficiency of the variable-time impulsive controller.
\end{abstract}

\section{Introduction}

Multiagent systems consist of many interacting dynamical units with specific contents $[1,2]$. They are wildly applied to both science and engineering fields like ecosystems, social networks, traffic management, expert systems, and so on. Recently, coordination phenomena in multiagent systems are investigated from a wide range of disciplines for their vast potential applications, including network management, wireless sensor networks, and autonomous vehicles maneuvering [3]. Among the common coordination phenomena such as synchronization and consensus of multiagent systems, coordinated tracking is an important topic. In this topic, a small number of agents play the leading roles in the systems, while others are the followers tracking the leader agents [4-6]. And the primary researches on coordinated tracking problem are to design appropriate control protocol for the multiagent system.

A lot of useful coordinated tracking control methods have been obtained in the previous research works. In [3], a constant bearing strategy was considered in the distributed control law for multiple autonomous surface vehicles. Adaptive neural network control of uncertain dynamical was studied in [7]; therein, the adaptive controller with an augmented
$\mathrm{NN}$ adaptive term was designed based on the state information of its neighborhood. And, moreover, this method was also extended to output feedback case. In [8], a nonlinear distributed control protocol for leader-following consensus of nonlinear multiagent was proposed. Fuzzy observed-based adaptive controller is proposed for second-order multiagent systems with heterogeneous nonlinear dynamics in [9]. It observes that all the control strategies in above-mentioned works were continuous time control. In realistic application, due to limited energy of agent and real-time communication constraints of networks, continuous time control for multiagent systems is generally costly. However, impulsive control provides the viewpoint that control strategy only occurs at some discrete times, which reduces the transmission among agents dramatically [10]. Because of the discretetime impulse, impulsive control protocol provides an efficient mechanism to deal with the large uncertainties in multiagent systems and has better performance in transient response and bandwidth usage.

Impulsive control for multiagent systems has been profoundly studied in many aspects, for example, network topology switch [11], different order agents [12], and communication time delay. In [11], a distributed impulsive protocol was proposed to implement second-order multitracking task 
by using only position sampled data of agents. Reference [13] investigated network-based leader-following impulsive consensus in nonlinear multiagent systems, which took networkdeduced delay into consideration. However, impulsive control of [11-13] often happens at fixed time, which means the impulsive instants are predesigned and independent of systems. In the practical cases, especially in biological and physiological fields, impulses do not always happen at fixed time. Instead, variable-time impulses often arise naturally. So, variable-time impulsive control for multiagent systems is much more practical in modeling and application. Despite these efforts on fixed-time impulsive control, there are few (if any) literatures addressing variable-time impulsive control for multiagent systems, because they present numerous analytical challenges of augmenting an variable-time impact on impulsive instants in impulsive control method.

On the other hand, a number of useful results have been obtained on variable-time impulsive system. In $[14,15]$, Bequivalence method was proposed to analyze discontinuous system. By using this method, variable-time impulsive system can be reduced to fixed-time one, which can be regarded as the comparison system of the original one. Based on these results, a theoretical framework was formulated to reveal the principle of reduction and comparison in nonlinear variable-time impulsive system in [16]. The authors therein constructed the relationship between the original jump operator and comparison system jump operator and then demonstrated that the two systems have the same stability property. The stability of Hopfield neural networks (HNN) with state-dependent impulses was discussed in [17] and the periodicity and stability for variable-time impulsive neural networks was discussed in [18]. Mittag-Leffler stability in fractional-order neural networks under time-varying control was investigated in $[19,20]$. In [21], the authors analyzed synchronization between two memristive systems under state-dependent control. Note that most of the existing results on stability discussion of variable-time impulsive system concentrated on system with one dynamic without communication topology. However, compared with systems with one or two dynamics, multiagent systems are composed of large number of agent dynamics and each agent interacts with other agents according to their communication topology. Therefore, the discussion on variable-time impulsive control for multiagent systems is more complex than the works mentioned above.

Motivated by the above discussions, we investigate variable-time impulsive control for coordinated tracking problem in nonlinear multiagent systems. The main contributions of this paper are twofold: first, compared with fixed-time impulsive control for coordinated tracking in multiagent systems in the existing works in [11-13], the impulsive time instants are designed to a more general case, varying with time, called variable-time impulsive control. To cope with the difficulty in directly analyzing the variable-time impulsive controllers, we select some proper conditions that guarantee that the coordinated tracking solution intersects impulsive surface only at once, and then B-equivalence method is employed to construct comparison systems with fixed-time impulsive controller of the variable-time ones; at last, we theoretically present that the two systems have the same stability. Coordinated tracking criteria of variabletime impulsive controller can be derived by analyzing fixedtime impulsive one without any difficulty. Second, from the variable-time impulsive system [16-21] standpoint, we extend the application of B-equivalence method on one dynamic to multiple dynamics, which should consider the constrains of communication topology among dynamics. And, moreover, coordinated tracking controller is designed to specific function containing impulsive matrices; this is more challenging in analysis than virtual functions in [16-21].

The rest of this paper is organized as follows: some preliminaries are introduced in Section 2. In Section 3, problem formulation is described and a global tracking error system between leader and followers is established. In Section 4, along the line of research on variable-time impulsive nonlinear system, some assumptions are proposed to ensure that each solution of the global tracking error system meets each surface of the discontinuity exactly once; then B-map between the original system and comparison system is constructed. According to the B-map and using Bequivalence method, original variable-time impulsive system is reduced to fixed-time one. Furthermore, we demonstrate that the two systems have the same stability properties. In Section 5, several sufficient criteria are proposed to guarantee the stability of the global tracking error system between the leader and the followers. By considering the selected Lyapunov function candidate and numerical simulation, we prove the followers' coordinated tracking of the leader under the variable-time impulsive control with the proposed criteria.

\section{Preliminaries}

In this section, some preliminaries about model are described. Let $G=(V, \zeta, A)$ be digraph (or directed graph) of order $N$ with a node set $V=\left\{\pi_{1}, \pi_{2} \cdots, \pi_{N}\right\}$ and an edge set $\zeta \subseteq V \times V$. The matrix $A=\left(a_{i j}\right)_{N \times N}$ is the weighted adjacency matrix; if there is connection between $\pi_{i}$ and $\pi_{j}, a_{i j}=1$; otherwise, $a_{i j}=0$. The index set of neighbors of node $\pi_{i}$ is denoted by $N_{i}=\left\{\pi_{i} \in V \mid\left(\pi_{j}, \pi_{i}\right) \in \zeta\right\}$. $D=\operatorname{diag}\left(d_{1}, d_{2}, \ldots, d_{N}\right)$, with $d_{i}=\sum_{j=1}^{n} a_{i j}$, denotes the degree matrix of graph $G$, and the Laplacian of graph $G$ is $L=D-A, L=\left(l_{i j}\right)_{N \times N}$. The Euclidean norm of a vector $x=\left(x_{1}, x_{2} \cdots, x_{n}\right)^{T} \in \mathbb{R}^{n}$ is denoted by $\|x\|=\sqrt{\sum_{i=1}^{n} x_{i}^{2}}$, and the spectral norm of a matrix $P=\left(p_{i j}\right)_{n \times n} \in \mathbb{R}^{n \times n}$ is denoted by $\|P\|=\sqrt{\lambda_{\max }\left(P^{T} P\right)} ; \lambda_{\max }(\cdot)$ is the maximum eigenvalue of matrix $P$. The appropriate dimension identity matrix is denoted by $I$. The Kronecker product of two matrices $R=\left(r_{i j}\right)_{n \times n}$ and $S=\left(s_{i j}\right)_{n \times n}$ is defined as

$$
R \otimes S=\left[\begin{array}{cccc}
r_{11} S & r_{12} S & \cdots & r_{1 n} S \\
r_{21} P & r_{22} S & \cdots & r_{2 n} S \\
\vdots & \vdots & \vdots & \vdots \\
r_{m 1} P & r_{m 2} S & \cdots & r_{m n} S
\end{array}\right],
$$


and some properties of Kronecker product are restated as follows:

$$
\begin{aligned}
\text { (i) } & k(R \otimes S)=k R \otimes S=R \otimes k S, \quad k \in \mathbb{R} ; \\
\text { (ii) } & (R+S) \otimes C=R \otimes C+S \otimes C ; \\
\text { (iii) } & (R \otimes S \otimes C)=R \otimes(S \otimes C) .
\end{aligned}
$$

\section{Problem Description}

Now we consider a class of multiagent systems consisting of $N$ follower agents indexed by $i \in\{1,2, \ldots, N\}$ and one leader agent labeled as $i=0$. The dynamics of $i$ th follower agent are governed by the following nonlinear dynamical equation:

$$
\dot{x}_{i}(t)=f\left(x_{i}(t)\right)+u_{i}(t)
$$

where $x_{i}=\left(x_{i 1}, x_{i 2}, \ldots, x_{i n}\right)^{T} \in \mathbb{R}^{n}$ is the state of agent $i$, $u_{i} \in \mathbb{R}^{n}$ denotes the controller of follower agent $i$, and $f\left(t, x_{i}\right)$ is continuous with $f(t, 0)=0$, which satisfies the Lipschitz condition with respect to $x$; that is, there exists a positive number $l_{f}$ such that $\left\|f\left(x_{i}\right)-f\left(x_{j}\right)\right\| \leq l_{f}\left\|x_{i}-x_{j}\right\|$ for any $x_{i}, x_{j} \in \mathbb{R}^{n}$.

The dynamics of the leader agent are described by

$$
\dot{x}_{0}(t)=f\left(x_{0}(t)\right),
$$

where $x_{0}$ is the state of the leader agent.

Make the assumption that at least one follower can exchange information with the leader. Then the impulsive coordinated tracking controller for multiagent systems is described as follows:

$$
\begin{aligned}
u_{i} & =\sum_{k=1}^{\infty} h\left(t-t_{k}\right) b_{i}\left(\left(\sum_{j \in N_{i}} a_{i j}\left(x_{i}(t)-x_{j}(t)\right)\right)\right. \\
& \left.+c_{i}\left(x_{i}(t)-x_{0}(t)\right)\right),
\end{aligned}
$$

where $t_{k}(k=1,2,3, \cdots)$ are the discrete instants varying with state of agent to be specified later; $h(t)$ is Dirac delta function with the property that $\int_{\alpha-\varepsilon}^{\alpha+\varepsilon} g(t) h(t-\alpha) d t=g(\alpha)$ for $\varepsilon \neq 0$. The Dirac delta function is often used to describe the narrow spike function such as an impulse. $b_{i} \in$ $\mathbb{R}^{n}$ where $b_{i}$ is the impulsive strength constant of the $i$ th controller to be designed later; $a_{i}$ is the connected weight between followers; if and only if there is an information communication between follower agents, $a_{i}=1$; otherwise $a_{i}=0, A=\operatorname{diag}\left(a_{1}, a_{2}, \ldots, a_{N}\right) \in \mathbb{R}^{N \times N}$, and $c_{i}$ is the connected weight between follower $x_{i}$ and leader $x_{0}$, similarly defined as $a_{i}, C=\operatorname{diag}\left(c_{1}, c_{2}, \ldots, c_{N}\right) \in \mathbb{R}^{N \times N}$. Without loss of generality, we assume that the solution $x_{i}(t)$ is leftcontinuous; that is, $\lim _{t \rightarrow \xi-0} x(t)=x(\xi)$.

In this paper, we design the impulsive instants varying with state of agent. This is different from the fixed-time impulsive controller designed in the previous works. The instants of impulsive controller adjust according to the agents' state; it is more realistic modeling in the field of ecological management and medical science. Moreover, we need not predesign the accurate impulsive instants before coordinated tracking. The function of instants $t_{k}$ is described as follows:

$$
t_{k}=\theta_{k}+\bar{\tau}_{k}(x),
$$

where $x=\left(x_{1}{ }^{T}, x_{2}{ }^{T}, \ldots, x_{N}{ }^{T}\right)^{T}, \theta_{k}$ satisfies $0=\theta_{0} \leq \theta_{1} \leq$ $\cdots \leq \theta_{k-1} \leq \theta_{k}<\cdots, \lim _{k \rightarrow+\infty} \theta_{k}=+\infty$, and $\bar{\tau}(x) \in \mathbb{R}$ is a continuous function.

Under variable-time impulsive controller, we say that followers coordinately tracked the leader if and only if

$$
\lim _{t \rightarrow \infty}\left\|x_{i}(t)-x_{0}(t)\right\|=0 .
$$

To move on, let $\delta_{i}(t)=x_{i}(t)-x_{0}(t)$ be the tracking error between follower $x_{i}(t)$ and leader $x_{0}(t), \delta=$ $\left(\delta_{1}{ }^{T}, \delta_{2}{ }^{T}, \ldots, \delta_{N}{ }^{T}\right)^{T}, x=\left(x_{1}{ }^{T}, x_{2}{ }^{T}, \ldots, x_{N}{ }^{T}\right)^{T}$, and $\mathbf{1}_{N}=$ $(I, I, \ldots, I)^{T}$; then the global tracking error $\delta(t)$ is described as

$$
\delta(t)=x-\mathbf{1}_{N} x_{0} .
$$

Denote $\Phi_{i}\left(\delta_{i}\right)=\Phi_{i}\left(x_{i}-x_{0}\right)=f\left(x_{i}\right)-f\left(x_{0}\right)$. Under controller (5), the tracking error dynamic can be written as an impulsive system:

$$
\begin{aligned}
& \dot{\delta}_{i}(t)=\Phi_{i}\left(\delta_{i}(t)\right), \quad t \neq \theta_{k}+\bar{\tau}_{k}(x), \\
& \Delta \delta_{i}(t) \\
& =b_{i}\left(\sum_{j \in N_{i}}\left(a_{i j}\left(x_{i}(t)-x_{j}(t)\right)\right)+c_{i}\left(x_{i}(t)-x_{0}(t)\right)\right), \\
& t=\theta_{k}+\bar{\tau}_{k}(x) .
\end{aligned}
$$

Let impulsive strength $b_{i}$ of each agent be the same constant that $b_{i}=b$; then system (9) can be written as a compact form:

$$
\begin{aligned}
\dot{\delta}(t) & =\Phi(\delta(t)), \quad t \neq \theta_{k}+\tau_{k}(\delta), \\
\Delta \delta(t) & =b((L+C) \otimes I) \delta(t), \quad t=\theta_{k}+\tau_{k}(\delta),
\end{aligned}
$$

where $\Phi(\delta)=\left(\Phi_{1}\left(\delta_{1}\right), \Phi_{2}\left(\delta_{2}\right), \ldots, \Phi_{N}\left(\delta_{N}\right)\right)^{T}, L$ is the Laplacian of communication graph of followers, $\tau(\delta) \in \mathbb{R}$ is a map of $\tau(x)$ satisfying $\tau(\delta)=\bar{\tau}(x)$ for all $x \in \mathbb{R}^{N \cdot n}$, $\tau_{i}(0)=0$, and there exists a positive number $l_{\tau}$ such that $\left\|\tau_{i}\left(\delta_{1}\right)-\tau_{i}\left(\delta_{2}\right)\right\| \leq l_{\tau}\left\|\delta_{1}-\delta_{2}\right\|$, for all $i \in \mathbb{Z}_{+}$. Moreover, we assume that there exists a positive constant $\varsigma$ such that $0 \leq \tau_{i}(\delta)<\varsigma$, for all $i \in \mathbb{Z}_{+}, \delta \in \mathbb{R}^{N \cdot n}$.

To move on, the following definitions are required.

Definition 1 (see [10]). Let $V: \mathbb{R}_{+} \times \mathbb{R}^{n \times N} \longrightarrow \mathbb{R}_{+}$; then $V$ is said to belong to class of $\sum$, if

(a) $V$ is continuous in $\left(\tau_{(i-1)}, \tau_{i}\right] \times \mathbb{R}^{n \times N}$ and, for each $\delta \epsilon$ $\mathbb{R}^{n \times N}, i=1,2, \ldots, \lim _{(t, y) \rightarrow\left(\tau_{i}^{+}, \delta\right)} V(t, y)=V\left(\tau_{i}^{+}, \delta\right)$ exists,

(b) $V$ is locally Lipschitzian in $\delta$.

According to the definition of $V$, it observes that, in analyzing the stability of ODE, $V$ associated with system (10) is the analog Lyapunov-like functions, which are usually discontinuous. Therefore, a generalized derivative known as the right and upper Dini's derivative should be defined. 
Definition 2 (see [10]). The right upper Dini's derivative of $V(t, \delta)$ is defined as

$$
\begin{aligned}
& D^{+} V(t, \delta) \\
& \quad \equiv \lim _{h \rightarrow 0^{+}} \sup \frac{1}{h}\{V[t+h, \delta+h \Phi(t, \delta)]-V(t, \delta)\} .
\end{aligned}
$$

Definition 3 (see [10]). For any $t \geq t_{0}$, if there exist constants $\alpha>0$ and $\omega>0$ such that the global tracking error

$$
\left\|\delta\left(t, t_{0}, \delta\left(t_{0}\right)\right)\right\| \leq \omega \exp \left(-\alpha\left(t-t_{0}\right)\right),
$$

system (10) is said to be exponentially stable.

From the definition of $\delta(t)$, one can find if system (10) is exponentially stable, the tracking error between followers and leader asymptotically reaches zero. For system (3), the followers exponentially coordinately tracked leader under controllers (5).

\section{Comparison System}

In this section, we shall construct a fixed-time impulsive system that can be considered as the comparison system of system (10) by using B-equivalence method. By means of B-equivalence method, we first propose some conditions that guarantee that each solution of system (10) meets the impulsive surface exactly at once and then prove that the two systems have the same stability.

Denote by $\Gamma_{i}=\left\{(t, \delta(t)) \in \mathbb{R}_{+} \times \mathbb{R}^{n \cdot N}: t=\theta_{i}+\tau_{i}(\delta(t)), t \in\right.$ $\left.\mathbb{R}_{+}, \delta \in \mathbb{R}^{n \cdot N}\right\}$ the $i$ th surface of discontinuity. By means of Bequivalence method, the following theorem is necessary.

Theorem 4. Each solution $\delta(t)$ of system (10) intersects each surface $\Gamma_{i}$ exactly once, if the following conditions hold:

(H1) There exist positive numbers $\underline{\theta}$ and $\bar{\theta}$, such that $\underline{\theta}+$ $\varsigma<\theta_{i}-\theta_{i-1}<\bar{\theta}-\varsigma$, for all $i \in \mathbb{Z}_{+}$, which demonstrates that $\underline{\theta}<\left[\theta_{i+1}+\tau_{i+1}(\delta)\right]-\left[\theta_{i}+\tau_{i}(\delta)\right]<\bar{\theta}$ and implies that "beating phenomenon" will not occur.

(H2) $\tau_{i}(\delta+b((L+C) \otimes I) \delta) \leq \tau_{i}(\delta)$, for all $i \in \mathbb{Z}_{+}$.

(H3) $\sqrt{N} l_{\tau} \cdot M_{\Phi}<1$, where $M_{\Phi}=\sup \left\|\Phi_{i}\left(t, \delta_{i}\right)\right\|<+\infty$.

We have the observations that if $\mathrm{H} 1$ holds, for $(i<j<k)$, $i \in \mathbb{Z}_{+}$, then each solution $\delta(t)$ of (10) intersects $\Gamma_{i}$ and $\Gamma_{k}$ must intersect $\Gamma_{k}$ and intersect every surface $\Gamma_{i}$; if $\mathrm{H} 2$ and $\mathrm{H} 3$ hold, each solution $\delta(t)$ of (10) intersects $\Gamma_{i}$ at most once. This proof follows theorem 1 in [16]; we omit it.

Now we shall use B-equivalent method to construct the comparison system of system (10).

Fix a number $i$; let $\delta^{0}(t)=\delta\left(t, \theta_{i}, \delta\right)$ be a solution of the continuous subsystem of (10) in time interval $\left[\theta_{i}, \xi_{i}\right]$, which intersects the surface $\Gamma_{i}$ of discontinuity at the moment $\xi_{i}$, and $\xi_{i}=\theta_{i}+\tau_{i}\left(\delta^{0}\left(\xi_{i}\right)\right)$. Note that $\xi_{i} \geq \theta_{i}$, because of $0 \leq \tau_{i}(\delta)<\varsigma$. Let $\delta^{1}(t)$ be another solution of the continuous subsystem of (10) in time interval $\left(\theta_{i}, \xi_{i}\right]$ having the relationship of $\delta^{0}(t)$ that

$$
\delta^{1}\left(\xi_{i}\right)=\delta^{0}\left(\xi_{i}+\right)=\delta^{0}\left(\xi_{i}\right)+b\left((L+C) \otimes I_{n}\right) \delta^{0}\left(\xi_{i}\right)
$$

Define the following map:

$$
\begin{aligned}
W_{i}(\delta) & =\delta^{1}\left(\theta_{i}\right)-\delta^{0}\left(\theta_{i}\right) \\
= & \delta^{1}\left(\xi_{i}\right)+\int_{\xi_{i}}^{\theta_{i}} \Phi\left(\delta^{1}(s)\right) d s-\delta^{0}\left(\theta_{i}\right) \\
= & \delta^{0}\left(\xi_{i}\right)+b\left((L+C) \otimes I_{n}\right) \delta^{0}\left(\xi_{i}\right) \\
& +\int_{\xi_{i}}^{\theta_{i}} \Phi\left(\delta^{1}(s)\right) d s-\delta^{0}\left(\theta_{i}\right) \\
= & \int_{\theta_{i}}^{\xi_{i}} \Phi\left(\delta^{0}(s)\right) d s \\
& +b\left((L+C) \otimes I_{n}\right)\left(\delta^{0}\left(\theta_{i}\right)+\int_{\theta_{i}}^{\xi_{i}} \Phi\left(\delta^{0}(s)\right) d s\right) \\
& +\int_{\xi_{i}}^{\theta_{i}} \Phi\left(\delta^{1}(s)\right) d s .
\end{aligned}
$$

Based on the map $W_{i}(\delta)$ and Figure 1, the following observations are obtained obviously.

Observation 5. $\delta^{0}(t)=\delta\left(t, \theta_{i}, \delta\right)$ can be extended to be system (10) in $\mathbb{R}_{+}$.

Observation 6. $\delta^{1}(t)=\delta\left(t, \xi_{i}, \delta^{0}\left(\xi_{i}+\right)\right)$ is the solution of the following multiagent systems with fixed-time impulsive control in $\mathbb{R}_{+}$:

$$
\begin{aligned}
\dot{\delta}(t) & =\Phi(t, \delta(t)), \quad t \neq \theta_{i}, \\
\Delta \delta & =W_{i}\left(\delta\left(\theta_{i}\right)\right), \quad t=\theta_{i} .
\end{aligned}
$$

Observation 7 . In the time interval $\left(\xi_{i-1}, \theta_{i}\right]$, for $i \in \mathbb{Z}_{+}$, one has

$$
\begin{aligned}
\delta^{0}(t) & \equiv \delta^{1}(t) \\
\delta^{1}\left(\theta_{i}+\right) & \equiv \delta^{0}\left(\theta_{i}\right)+W_{i}\left(\delta^{0}\left(\theta_{i}\right)\right) \\
\delta^{1}\left(\xi_{i}+\right) & =\delta^{0}\left(\xi_{i}\right)+b_{k}(L+C) \delta^{0}\left(\xi_{i}\right) .
\end{aligned}
$$

Observation 8. For all $i \in \mathbb{Z}_{+}$, on time interval $\left(\theta_{i}, \xi_{i}\right]$,

$$
\begin{aligned}
\delta^{1}(t)-\delta^{0}(t)= & \delta^{0}\left(\theta_{i}\right)+W_{i}\left(\delta^{0}\left(\theta_{i}\right)\right) \\
& +\int_{\theta_{i}}^{t} \Phi\left(\delta^{1}(u)\right) d u-\delta^{0}\left(\theta_{i}\right) \\
& -\int_{\theta_{i}}^{t} \Phi\left(\delta^{0}(u)\right) d u \\
= & W_{i}\left(\delta^{0}\left(\theta_{i}\right)\right) \\
& +\int_{\theta_{i}}^{t}\left(\Phi\left(\delta^{1}(u)\right)-\Phi\left(\delta^{0}(u)\right)\right) d u .
\end{aligned}
$$

To present the relationship of system (15) and system (10), we have the following theorem. 


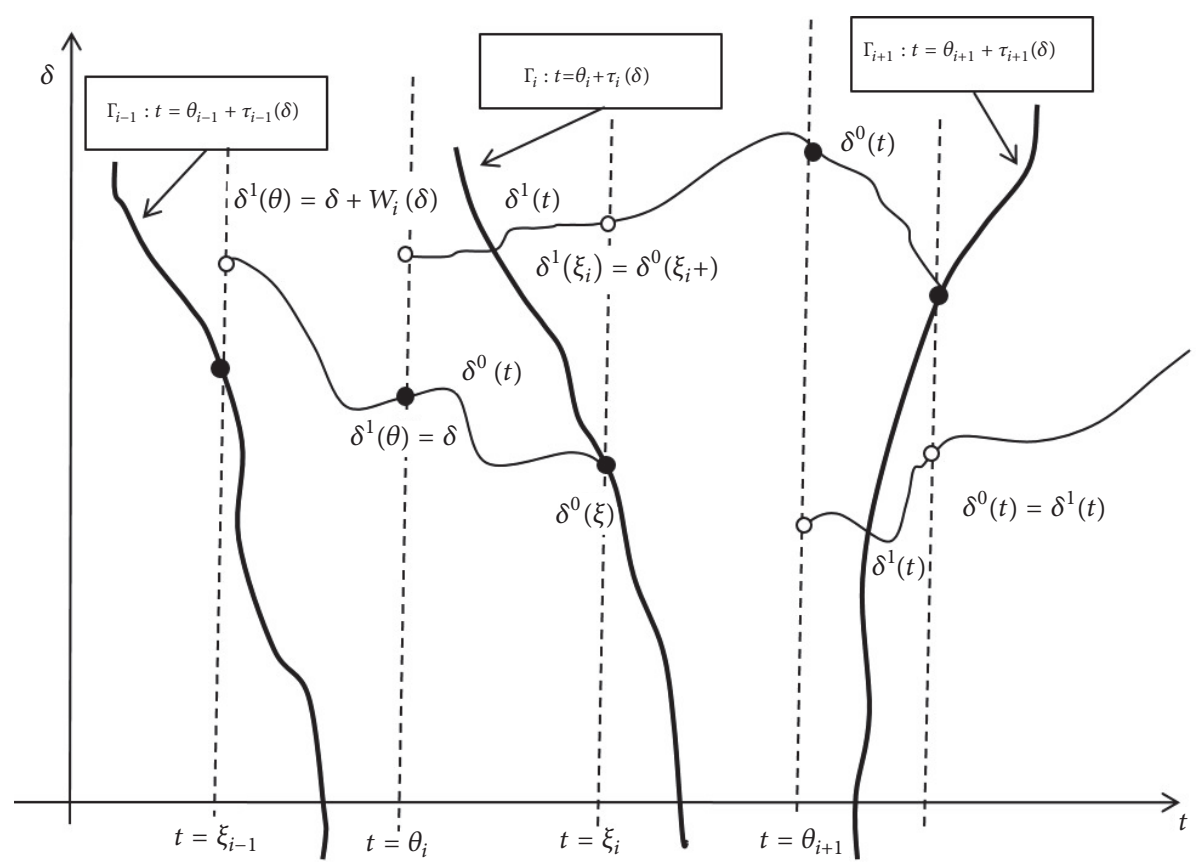

FIgure 1: The $B-$ map of the two systems.

Theorem 9. The variable-time impulsive system (10) and the fixed-time impulsive system (15) have the same stability property.

Proof. From the definition of $W_{i}\left(\delta^{0}\left(\theta_{i}\right)\right)$, we have estimation of $\left\|W_{i}\left(\delta^{0}\left(\theta_{i}\right)\right)\right\|$ as follows:

$$
\begin{aligned}
& \left\|W_{i}\left(\delta^{0}\left(\theta_{i}\right)\right)\right\|=\| \int_{\theta_{i}}^{\xi_{i}} \Phi\left(\delta^{0}(s)\right) d s \\
& +b\left((L+C) \otimes I_{n}\right)\left(\delta^{0}\left(\theta_{i}\right)+\int_{\theta_{i}}^{\xi_{i}} \Phi\left(\delta^{0}(s)\right) d s\right) \\
& +\int_{\xi_{i}}^{\theta_{i}} \Phi\left(\delta^{1}(s)\right) d s \| \leq 2 \sqrt{N} M_{\bar{\Phi}}\left(\xi_{i}-\theta_{i}\right) \\
& +\left\|b\left((L+C) \otimes I_{n}\right)\right\|\left\|\delta^{0}\left(\theta_{i}\right)\right\| \\
& +\sqrt{N} M_{\bar{\Phi}}\left\|b\left((L+C) \otimes I_{n}\right)\right\|\left(\xi_{i}-\theta_{i}\right)=\sqrt{N} M_{\bar{\Phi}}(2 \\
& \left.+b\left\|\left((L+C) \otimes I_{n}\right)\right\|\right)\left(\tau_{i}\left(\delta^{0}\left(\xi_{i}\right)\right)\right) \\
& +b\left\|\left((L+C) \otimes I_{n}\right)\right\|\left\|\delta^{0}\left(\theta_{i}\right)\right\| .
\end{aligned}
$$

Note that

$$
\begin{aligned}
\tau_{i}\left(\delta^{0}\left(\xi_{i}\right)\right) & \leq l_{\tau}\left\|\delta^{0}\left(\xi_{i}\right)\right\| \\
& =l_{\tau}\left\|\delta^{0}\left(\theta_{i}\right)+\int_{\theta_{i}}^{\xi_{i}} \Phi\left(\delta^{0}(s)\right) d s\right\|
\end{aligned}
$$

$$
\begin{aligned}
& \leq l_{\tau}\left\|\delta^{0}\left(\theta_{i}\right)\right\|+l_{\tau}\left\|\int_{\theta_{i}}^{\xi_{i}} \Phi\left(\delta^{0}(s)\right) d s\right\| \\
& \leq l_{\tau}\left\|\delta^{0}\left(\theta_{i}\right)\right\|+\sqrt{N} M_{\Phi} l_{\tau}\left(\xi_{i}-\theta_{i}\right) \\
& =l_{\tau}\left\|\delta^{0}\left(\theta_{i}\right)\right\|+\sqrt{N} M_{\Phi} l_{\tau} \tau_{i}\left(\delta\left(\xi_{i}\right)\right)
\end{aligned}
$$

That is,

$$
\tau_{i}\left(\delta^{0}\left(\xi_{i}\right)\right) \leq\left(1-\sqrt{N} M_{\Phi} l_{\tau}\right)^{-1} l_{\tau}\left\|\delta^{0}\left(\theta_{i}\right)\right\| .
$$

We get

$$
\begin{aligned}
& \left\|W_{i}\left(\delta^{0}\left(\theta_{i}\right)\right)\right\| \leq\left(\sqrt{N} M_{\Phi} l_{\tau}(2+b\|((L+C) \otimes I)\|)\right. \\
& \left.\cdot\left(1-\sqrt{N} M_{\Phi} l_{\tau}\right)^{-1}+b\|((L+C) \otimes I)\|\right)\left\|\delta^{0}\left(\theta_{i}\right)\right\| \\
& =\bar{d}_{k}\left\|\delta^{0}\left(\theta_{i}\right)\right\|,
\end{aligned}
$$

where $\bar{d}_{k}=\sqrt{N} M_{\Phi} l_{\tau}(2+b\|((L+C) \otimes I)\|)\left(1-\sqrt{N} l_{\tau} M_{\Phi}\right)^{-1}+$ $b\|((L+C) \otimes I)\|$.

Moreover, for all $t \in\left(\theta_{i}, \xi_{i}\right]$, we can obtain

$$
\begin{aligned}
\left\|\delta^{1}(t)-\delta^{0}(t)\right\| \leq & \left\|W\left(\delta^{0}\left(\theta_{i}\right)\right)\right\| \\
& +\int_{\theta_{i}}^{t}\left\|\Phi\left(\delta^{1}(u)\right)-\Phi\left(\delta^{0}(u)\right)\right\| d u \\
\leq & \left\|W\left(\delta^{0}\left(\theta_{i}\right)\right)\right\| \\
& +l_{\Phi} \int_{\theta_{i}}^{t}\left\|\delta^{1}(u)-\delta^{0}(u)\right\| d u,
\end{aligned}
$$


and by utilizing the Gronwall-Bellman Inequality, one has

$$
\begin{aligned}
\left\|\delta^{1}(t)-\delta^{0}(t)\right\| & \leq\left\|W\left(\delta^{0}\left(\theta_{i}\right)\right)\right\| e^{l_{\Phi}\left(t-\theta_{i}\right)} \\
& \leq \bar{d}_{k} e^{l_{\Phi} \varsigma}\left\|\delta^{0}\left(\theta_{i}\right)\right\|
\end{aligned}
$$

This completes the proof.

From Theorem 9, system (15) can be regarded as the comparison system of system (10). Therefore, we can get the stability criteria of system (10) by analyzing system (15). Thus, it avoids difficulty in directly considering variable-time impulsive system.

\section{Main Results}

We know that the impulsive system (10) is the compact global tracking error form of multiagent systems (3) and (4) with impulsive control instants function (5). The stability criteria of system (10) can be obtained by considering system (15). Therefore, we proposed the following theorem.

Theorem 10. The comparison system (15) is globally exponentially stable, if there exists a positive constant $\gamma$ such that

$$
\ln d_{k}^{2}+l_{f}(\bar{\theta}-\varsigma) \leq-\gamma
$$

where $_{k}=\sqrt{N} M_{\Phi} l_{\tau}(2+\|b((L+C) \otimes I)+I\|)\left(1-\sqrt{N} l_{\tau} M_{\Phi}\right)^{-1}+$ $b\|((L+C) \otimes I)+I\|$.

Before the proof of this theorem, we first compute the range of $\left\|W_{i}(\delta)+\delta\right\|$; similar to computation of $\left\|W_{i}(\delta)\right\|$ in Section 4 , we have

$$
\begin{aligned}
& \left\|W_{i}\left(\delta^{0}\left(\theta_{i}\right)\right)+\delta^{0}\left(\theta_{i}\right)\right\| \\
& \leq\left(\sqrt{N} M_{\Phi} l_{\tau}(2+\|b((L+C) \otimes I)\|)\right. \\
& \left.\cdot\left(1-\sqrt{N} l_{\tau} M_{\Phi}\right)^{-1}+\|b((L+C) \otimes I)+I\|\right) \\
& \cdot\left\|\delta^{0}\left(\theta_{i}\right)\right\| .
\end{aligned}
$$

Proof. The following Lyapunov function is considered:

$$
V(\delta)=\delta^{T}(t) \delta(t)
$$

When $t \neq \theta_{k}$, by calculating the Dini derivative of $V(\delta)$ along the continuous subsystem of (15), we can obtain that

$$
\begin{aligned}
& D^{+} V(\delta(t))=2 \delta(t)^{T} \Phi(\delta(t)) \\
& \quad=\sum_{i=1}^{N} \delta_{i}(t)\left(f\left(x_{i}(t)\right)-f\left(x_{0}(t)\right)\right) \\
& \quad \leq \sum_{i=1}^{N}\left(x_{i}(t)-x_{0}(t)\right)\left(f\left(x_{i}(t)\right)-f\left(x_{0}(t)\right)\right) \\
& \quad \leq l_{f} \sum_{i=1}^{N}\left\|x_{i}(t)-x_{0}(t)\right\|^{2} \leq l_{f} \delta(t)^{T} \delta(t) .
\end{aligned}
$$

When $t=\theta_{k}$, it follows from the state jump subsystem of (15) and (25) that

$$
\begin{aligned}
V & \left(\delta\left(\theta_{k}\right)+W_{k}\left(\delta\left(\theta_{k}\right)\right)\right) \\
& =\left[\delta\left(\theta_{k}\right)+W_{k}\left(\delta\left(\theta_{k}\right)\right)\right]^{T}\left[\delta\left(\theta_{k}\right)+W_{k}\left(\delta\left(\theta_{k}\right)\right)\right] \\
& =\left\|\delta\left(\theta_{k}\right)+W_{k}\left(\delta\left(\theta_{k}\right)\right)\right\|^{2} \leq d_{k}^{2}\left\|\delta\left(\theta_{k}\right)\right\|^{2} \\
& =d_{k}^{2}\left(\left(\delta\left(\theta_{k}\right)\right)^{T}\left(\delta\left(\theta_{k}\right)\right) .\right.
\end{aligned}
$$

Therefore, we have

$$
\begin{gathered}
D^{+} V(\delta(t)) \leq l_{f} V(\delta(t)), \quad t \neq \theta_{k}, \\
V\left(\delta\left(\theta_{k}\right)+W_{k}\left(\delta\left(\theta_{k}\right)\right)\right) \leq d_{k}^{2} V\left(\delta\left(\theta_{k}\right)\right), \quad t=\theta_{k} .
\end{gathered}
$$

We now show that the following claim holds.

Claim. $V(\delta(t)) \leq V_{0} \exp \left\{l_{f} t+\sum_{i=1}^{k} \ln d_{i}^{2}\right\}$, for any $t \in$ $\left(\theta_{k}, \theta_{k+1}\right], k \in \mathbb{Z}_{+}$, where $V_{0}=V(\delta(0))$.

We introduce the mathematical induction to prove the claim.

(i) When $t \in\left(0, \theta_{1}\right]$ and $k=0$, from (26) and (30), we have

$$
\begin{aligned}
& V(\delta(t)) \leq V_{0} \exp \left\{l_{f} t\right\}, \\
& V\left(\delta\left(\theta_{1}\right)+W_{1}\left(\delta\left(\theta_{1}\right)\right)\right) \leq d_{1}^{2} V\left(\delta\left(\theta_{1}\right)\right) \\
& \quad \leq d_{1}^{2} V_{0} \exp \left\{l_{f} \theta_{1}\right\} .
\end{aligned}
$$

(ii) When $t \in\left(\theta_{1}, \theta_{2}\right]$ and $k=1$, from (15), it follows that

$$
\begin{aligned}
& V(\delta(t)) \leq V\left(\delta\left(\theta_{1}+\right)\right) \exp \left\{l_{f} t\right\} \\
& \quad=V_{0} \exp \left\{l_{f} t+\ln d_{1}^{2}\right\}, \\
& V\left(\delta\left(\theta_{2}\right)+W_{2}\left(\delta\left(\theta_{2}\right)\right)\right) \leq d_{2}^{2} V\left(\delta\left(\theta_{2}\right)\right) \\
& \quad \leq d_{1}^{2} d_{2}^{2} V_{0} \exp \left\{l_{f} \theta_{2}\right\},
\end{aligned}
$$

and, therefore, when $t \in\left(\theta_{1}, \theta_{2}\right]$ and $k=1$, the claim is true.

(iii) When $k=s, s>1$, we assume that the claim holds; that is,

$$
V(\delta(t)) \leq V_{0} \exp \left\{l_{f} t+\sum_{i=1}^{s} \ln d_{i}^{2}\right\} \quad t \in\left(\theta_{s}, \theta_{s+1}\right] .
$$

(iv) When $t \in\left(\theta_{s+1}, \theta_{s+2}\right]$ and $k=s+1$, one observes that

$$
\begin{aligned}
V(\delta(t)) \leq & V\left(\delta\left(\theta_{s+1}\right)\right) \\
& +W_{s+1}\left(\delta\left(\theta_{s+1}\right)\right) \exp \left\{l_{f}\left(t-\theta_{s+1}\right)\right\} \\
\leq & d_{s+1}^{2} V_{0} \exp \left\{l_{f} t+\sum_{i=1}^{s} \ln d_{i}^{2}\right\} \\
= & V_{0} \exp \left\{l_{f} t+\sum_{i=1}^{s+1} \ln d_{i}^{2}\right\} .
\end{aligned}
$$


From the above discussion, when $t \in\left(\theta_{s+1}, \theta_{s+2}\right]$ and $k=s+1$, the claim holds. So, for all $t \in\left(\theta_{k}, \theta_{k+1}\right], k \in \mathbb{Z}_{+}$, the claim holds.

Note that

$$
\begin{aligned}
\theta_{k+1} & =\theta_{k+1}-\theta_{k}+\theta_{k}-\theta_{k-1}+\cdots+\theta_{2}-\theta_{1}+\theta_{1}-\theta_{0} \\
& \leq \sum_{i=1}^{k+1}\left(\theta_{i}-\theta_{i-1}\right) \leq(k+1)(\bar{\theta}-\varsigma) .
\end{aligned}
$$

Therefore, when $t \in\left(\theta_{k}, \theta_{k+1}\right]$, we have $t \leq(k+1)(\bar{\theta}-\varsigma)$, which implies that $k \geq t /(\bar{\theta}-\varsigma)-1$ and

$$
\begin{aligned}
& V(\delta(t)) \leq V_{0} \exp \left\{l_{f} t+\sum_{i=1}^{k} \ln d_{i}^{2}\right\} \leq V_{0} \\
& \cdot \exp \left\{l_{f} \sum_{i=1}^{k+1}\left(\theta_{i}-\theta_{i-1}\right)+\sum_{i=1}^{k} \ln d_{i}^{2}\right\}=V_{0} \\
& \cdot \exp \left\{l_{f}(\bar{\theta}-\varsigma)\right\} \exp \left\{\sum_{i=1}^{k}\left[\ln d_{i}^{2}+l_{f}\left(\theta_{i}-\theta_{i-1}\right)\right]\right\} \\
& \leq V_{0} \exp \left\{l_{f}(\bar{\theta}-\varsigma)\right\} \exp \{-k \gamma\}<V_{0} \\
& \cdot \exp \left\{l_{f}(\bar{\theta}-\varsigma)+\gamma\right\} \exp \left\{-\left(\frac{\gamma}{(\bar{\theta}-\varsigma)}\right) t\right\} .
\end{aligned}
$$

Hence, we can obtain from (26) that

$$
\begin{aligned}
& \|\delta(t)\| \\
& \quad \leq \sqrt{V_{0} \exp \left\{l_{f}(\bar{\theta}-\varsigma)+\gamma\right\}} \exp \left\{-\frac{\gamma t}{(2(\bar{\theta}-\varsigma))}\right\} .
\end{aligned}
$$

The last inequality implies that system (15) is globally exponentially stable.

Combining Definition 3 and Theorem 9, we have the following corollary without proof.

Corollary 11. Under the stability criteria of system (15), the followers of the multiagent systems coordinately track the leader by variable-time impulsive control.

\section{Numerical Simulation}

In this section, a numerical simulation is given to illustrate the correctness of the theoretical results obtained in the previous sections. Consider multiagent systems consisting of 3 followers and 1 leader agent with the communication

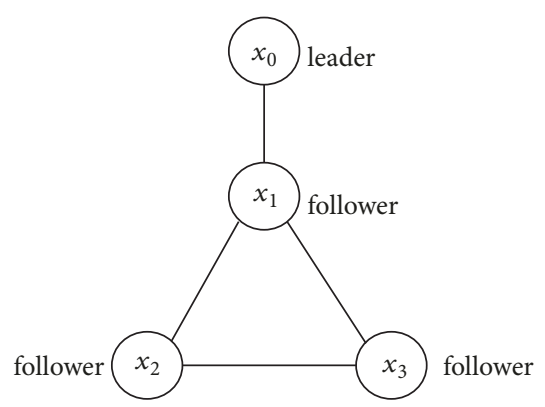

Figure 2: The topological structure of one leader and 3 followers.

topology represented by Figure 2. And the Laplacian and the connection matrix are defined as follows:

$$
\begin{aligned}
& L=\left[\begin{array}{ccc}
2 & -1 & -1 \\
-1 & 2 & -1 \\
-1 & -1 & 2
\end{array}\right], \\
& C=\left[\begin{array}{lll}
1 & 0 & 0 \\
0 & 0 & 0 \\
0 & 0 & 0
\end{array}\right] .
\end{aligned}
$$

The dynamics of each agent are governed by the Chua circuit [22], which exhibits the chaotic phenomena described as

$$
f\left(x_{i}((t))=\left\{\begin{array}{l}
-\alpha\left[x_{i 1}-x_{i 2}-\rho_{i 1}\left(x_{i 1}\right)\right] \\
x_{i 1}-x_{i 2}+x_{i 3} \\
-\beta x_{i 2}
\end{array}\right.\right.
$$

where $\left\|x_{i j}\right\| \leq 1, i=0,1,2,3, j=1,2,3, \alpha=9.2156, \beta=$ 15.9946, $\rho_{i 1}=p x_{i 1}+0.5(g-p)\left[\left\|x_{i 1}+1\right\|-\left\|x_{i 1}-1\right\|\right]$, and $g=-1.24905$ and $p=-0.75735$.

Equation (39) is rewritten into compact form as follows:

$$
f\left(x_{i}(t)\right)=Q x_{i}(t)+\rho\left(x_{i}(t)\right),
$$

where $x_{i}(t)=\left(x_{i 1}, x_{i 2}, x_{i 3}\right)^{T} \in \mathbb{R}^{3 \times 3}, Q=\left[\begin{array}{ccc}-\alpha(1+p) & \alpha & 0 \\ 1 & -1 & 1 \\ 0 & -\beta & 0\end{array}\right]$ and $\rho\left(\left(x_{i}(t)\right)\right)=\left[0.5(g-p)\left(\left\|x_{i 1}+1\right\|-\left\|x_{i 1}-1\right\|, 0.0\right)\right]^{T}$.

To make followers coordinately track the leader, we design the following impulsive controller with impulsive instants varying with error $\delta_{21}, t=0.01 k+0.0001\left\|\delta_{21}\right\|$, and $b=-0.36$.

$$
\begin{aligned}
u_{i}(t)= & \left.-0.36 \sum_{j \in N_{i}}\left(x_{i}(t)-x_{j}(t)\right)\right) \\
& +c_{i}\left(x_{i}(t)-\left.x_{0}(t)\right|_{t=0.01 k+0.0001\left\|\delta_{21}\right\|},\right.
\end{aligned}
$$


and, together with (40), the multiagent system under variable-time impulsive control can be described as

$$
\begin{array}{rl}
\dot{x}_{i}= & Q x_{i}+\rho\left(x_{i}\right), \quad t \neq 0.01 k+0.0001\left\|\delta_{21}\right\|, \\
\Delta x_{i}= & -0.36\left(\sum_{j \in N_{i}}\left(x_{i}(t)-x_{j}(t)\right)\right) \\
& +c_{i}\left(x_{i}(t)-x_{0}(t)\right), \\
t & t=0.01 k+0.0001\left\|\delta_{21}\right\| .
\end{array}
$$

By means of B-equivalence method of systems (42), (H1)(H3) in Theorem 4 should be satisfied.

For $(\mathrm{H} 1)$, note that $l_{\tau}=0.0001, \varsigma=\max \{\tau(\delta)\}=0.00036$, and $\theta_{i+1}-\theta_{i}=0.01$. Obviously, there exist positive numbers $\underline{\theta}$ and $\bar{\theta}$, such that $\underline{\theta}+\varsigma<\theta_{i}-\theta_{i-1}<\bar{\theta}-\varsigma$; that is, (H1) holds. have

For $(\mathrm{H} 2)$, note that $b=-0.36$ and $\tau(\delta)=0.0001\left\|\delta_{21}\right\|$; we

$$
\begin{aligned}
& \tau_{k}\left(\delta_{21}+b(L+C) \delta_{21}\right)-\tau_{k}\left(\delta_{21}\right) \\
& \quad \leq 0.0001\left\|I_{3}+b(L+C)\right\|\left\|\delta_{21}\right\|-0.0001\left\|\delta_{21}\right\| \\
& \quad=0.0001 \times\left(0.9035\left\|\delta_{21}\right\|-\left\|\delta_{21}\right\|\right) \leq 0,
\end{aligned}
$$

and, therefore, $\tau_{k}(\delta+b(L+C) \otimes I) \leq \tau_{k}(\delta)$; (H2) holds.

For conditions (H3), we have the following computation:

$$
\begin{aligned}
M_{\Phi} & =\sup \left\|f\left(x_{i}\right)-f\left(x_{0}\right)\right\| \\
& \leq \sup \left\|f\left(x_{i}\right)\right\|+\sup \left\|f\left(x_{0}\right)\right\| \\
& \leq 2 \sup \left\|Q x_{i}(t)+\rho\left(x_{i}(t)\right)\right\| \\
& \leq 2\left(\|Q\|\left\|x_{i}(t)\right\|+\left\|\rho\left(x_{i}\right)\right\|\right) \\
& \leq 2\left(\|Q\|\left\|x_{i}(t)\right\|+|g-p|\right)=65.0674,
\end{aligned}
$$

and thus $\sqrt{N} l_{\tau} M_{\Phi}=1.7321 \times 0.0001 \times 65.0674<1 ;(\mathrm{H} 3)$ holds.

Thereupon, (H1)-(H3) hold, which illustrate that the parameters we selected guarantee that solutions of systems (42) intersect the surface $t=0.01 k+0.0001\left\|\delta_{21}\right\|$ exactly at once. Therefore, the comparison systems associated with tracking error system of (42) are presented by employing Bequivalence method:

$$
\begin{aligned}
& \dot{\delta}_{i}(t)=Q x_{i}(t)+\rho\left(x_{i}(t)\right)-\left(Q x_{0}(t)+\rho\left(x_{0}(t)\right)\right), t \neq 0.01 k, \\
& \Delta \delta_{i}(t)=W\left(\delta_{i}(t)\right), \quad t=0.01 k,
\end{aligned}
$$

where $k \in \mathbb{Z}_{+} ; W\left(\delta_{i}(t)\right)$ is defined in Section 4 .

By utilizing the results of Theorem 10, we consider this Lyapunov function candidate of system (45):

$$
V(\delta(t))=\delta(t)^{T} \delta(t)
$$

As each agent governed by Chua circuit, when $t \neq 0.01 k$, the Dini derivative $V(\delta(t))$ with respect to time $t$ is calculated as follows:

$$
\begin{aligned}
& D^{+} V(\delta(t))=2 \sum_{i=1}^{i=3}\left(x_{i}(t)-x_{0}(t)\right)^{T} \\
& \cdot\left(Q x_{i}(t)+\rho\left(x_{i}(t)\right)-Q x_{0}(t)-\rho\left(x_{0}(t)\right)\right) .
\end{aligned}
$$

Noting that $g-p \leq 0$ and $-1 \leq x_{i j} \leq 1$,

$$
\begin{aligned}
& \left(x_{i}(t)-x_{0}(t)\right)^{T}\left(\rho\left(x_{i}(t)\right)-\rho\left(x_{0}(t)\right)\right) \\
& \quad=\sum_{i=0}^{3}\left(0.5(g-p)\left(x_{i 1}(t)-x_{01}(t)\right)\right. \\
& \left.\quad \cdot\left(\left\|x_{i 1}+1\right\|-\left\|x_{i 1}-1\right\|-\left\|x_{01}+1\right\|-\left\|x_{01}-1\right\|\right)\right) \\
& \quad=(g-p) \sum_{i=0}^{3}\left(x_{i 1}-x_{01}\right)^{2} \leq 0 .
\end{aligned}
$$

Then,

$$
\begin{aligned}
& D^{+} V(\delta(t)) \\
& \quad \leq 2 \sum_{i=1}^{i=3}\left(x_{i}(t)-x_{0}(t)\right)^{T}\left(Q x_{i}(t)-Q x_{0}(t)\right) \\
& \quad \leq \lambda_{\max }\left(Q+Q^{T}\right)\left(x_{i}(t)-x_{0}(t)\right)^{T}\left(x_{i}(t)-x_{0}(t)\right) \\
& \quad=\lambda_{\max }\left(Q+Q^{T}\right) \delta(t)^{T} \delta(t) \\
& \quad \leq 16.5498 \delta(t)^{T} \delta(t)
\end{aligned}
$$

When $t=0.01 k$, according to inequality (25), one has

$$
\begin{aligned}
V & (\delta(t)+W(\delta(t))) \\
& =[W(\delta(t))+\delta(t)]^{T}[W(\delta(t))+\delta(t)] \\
& \leq d_{k}{ }^{2} \delta(t)^{T} \delta(t)
\end{aligned}
$$

where $d_{k}=\sqrt{N} M_{\Phi} l_{\tau}(2+\|b((L+C) \otimes I)+I\|)\left(1-\sqrt{N} l_{\tau} M_{\Phi}\right)^{-1}+$ $b\|((L+C) \otimes I)+I\|$.

Substituting parameters to $d_{k}$, we calculate $d_{k}=0.9418$. Thus, we have $l_{f}=16.5498, d_{k}=0.9418$, and $\bar{\theta}-\varsigma=0.01$ of criteria in Theorem 10; then $\gamma=0.0746$, which satisfy criteria of Theorem 10. Therefore, system (45) is globally exponentially stable. Combining with Corollary 11, we have reasons to state that, under the variable-time controller (42), followers exponentially coordinately tracked the leader. Taking initial values of agents randomly selected as $x_{0}(0)=$ $(-0.0032,-0.4190,0.3455)^{T}$, the followers

$$
x_{i j}(0)=\left[\begin{array}{ccc}
0.9760 & 0.5331 & 0.3322 \\
-0.7381 & -0.8092 & -0.9703 \\
-0.4236 & 0.6335 & 0.9710
\end{array}\right] \text {, }
$$




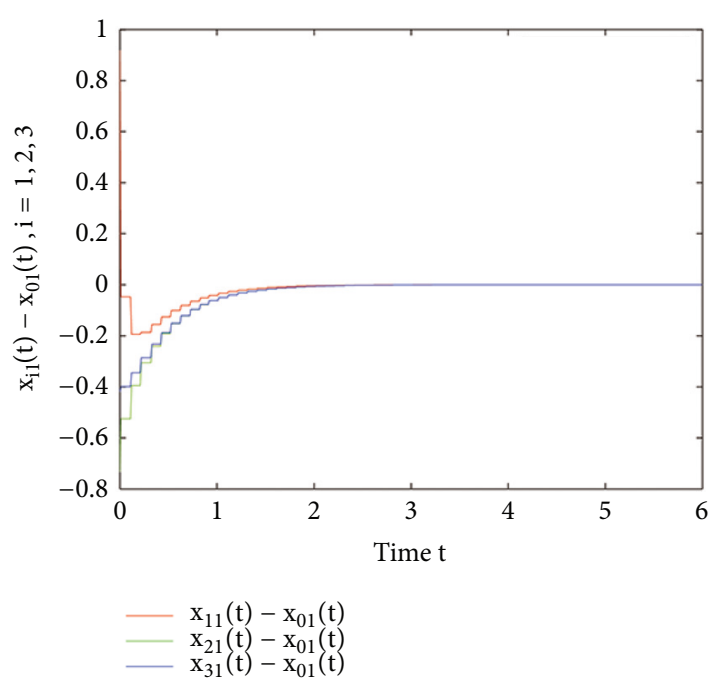

FIGURE 3: The 1-dimensional error value of agent $x_{i}$ and leader agent $x_{0}$.

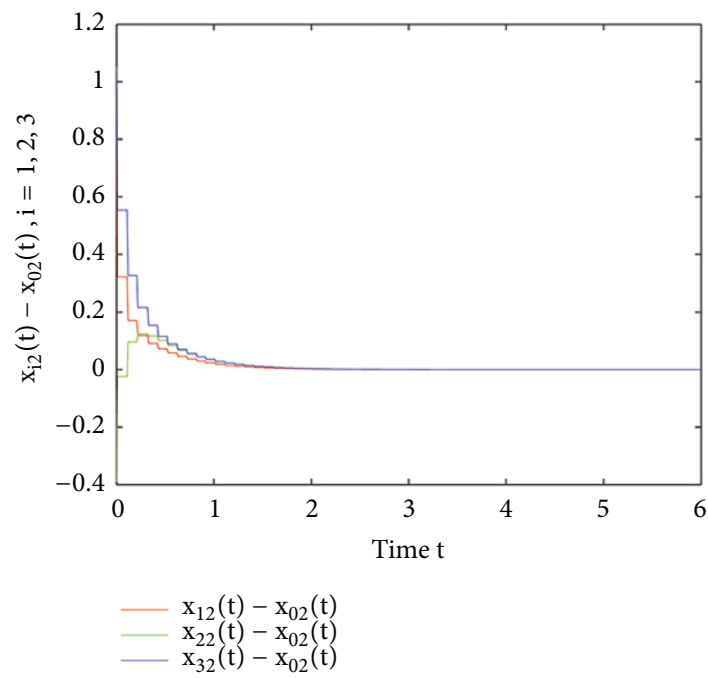

Figure 4: The 2-dimensional error value of agent $x_{i}$ and leader agent $x_{0}$.

where $x_{i j}$ denotes the initial value of $j$-dimensional agents $x_{i}(0), 1 \leq i \leq 3,1 \leq j \leq 3$.

Simulation results are shown in Figures 3-5. Figures 3, 4, and 5, respectively, show the 1-dimensional, 2-dimensional, and 3-dimensional tracking error between followers and leader. It can be seen from Figures 3-5 that each dimensional error between followers and leader converges to 0 . These illustrate that each follower coordinately tracked the leader under the variable-time impulsive control we proposed.

\section{Conclusion}

In this paper, we have investigated coordinated tracking problem in the nonlinear multiagent systems under variabletime impulsive control. Rigorous analysis by employing Bequivalence method has demonstrated that the variabletime impulsive controller we proposed guarantees that each

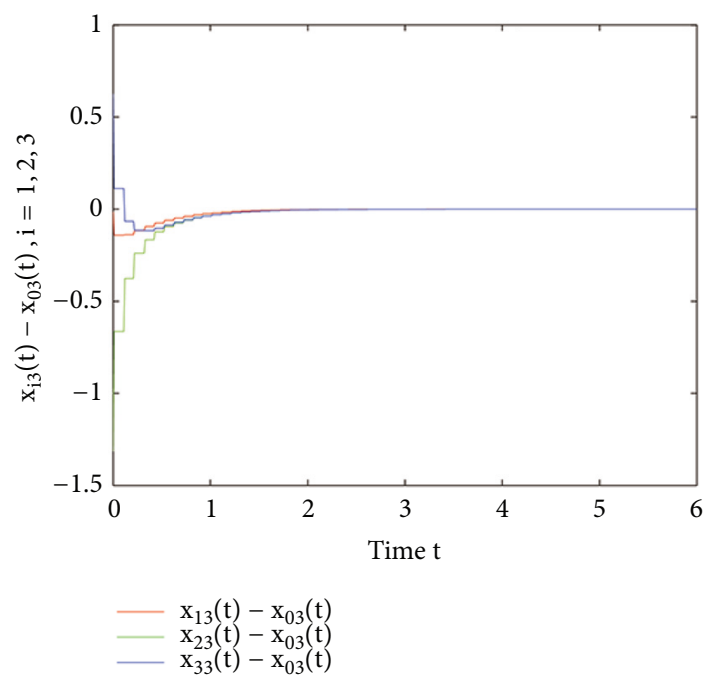

FIGURE 5: The 3-dimensional error value of agent $x_{i}$ and leader agent $x_{0}$.

follower agent coordinately tracked the leader. However, the constraint of impulsive instants function $\left.t_{k}=\theta_{k}+\tau_{(} \delta\right)$ is rigorous, and the stability criteria are also conservative as a result of conservative estimation on norm of map $W_{k}(\delta)$. It is excepted to extend this presented method to more general variable-time impulsive controller for multiagent systems.

\section{Data Availability}

The readers are welcome to request the source codes of the figures by sending an email to licd@cqu.edu.cn.

\section{Conflicts of Interest}

The authors declare that they have no conflicts of interest.

\section{Acknowledgments}

This work is supported by the National Natural Science Foundation of China (no. 61633011 and no. 61672119), the Qatar National Research Fund, a member of the Qatar Foundation, the National Priorities Research Program under Grant NPRP 9-166-1-031, and the Chongqing Research Program of Basic Research and Frontier Technology (cstc2015jcyjA40041 and cstc2015jcyjBX0052).

\section{References}

[1] X. Liu, K. Zhang, and W.-C. Xie, "Consensus seeking in multi-agent systems via hybrid protocols with impulse delays," Nonlinear Analysis: Hybrid Systems, vol. 25, pp. 90-98, 2017.

[2] W. Li, H. Zhou, Z.-W. Liu, Y. Qin, and Z. Wang, "Impulsive coordination of nonlinear multi-agent systems with multiple leaders and stochastic disturbance," Neurocomputing, vol. 171, pp. 73-81, 2016. 
[3] Z. Peng, J. Wang, and D. Wang, "Distributed maneuvering of autonomous surface vehicles based on neurodynamic optimization and fuzzy approximation," IEEE Transactions on Control Systems Technology, vol. 26, no. 3, pp. 1083-1090, 2018.

[4] J. Wang, "Distributed coordinated tracking control for a class of uncertain multiagent systems," IEEE Transactions on Automatic Control, vol. 62, no. 7, pp. 3423-3429, 2017.

[5] Y. Zhao, Z. Duan, G. Wen, and G. Chen, "Robust consensus tracking of multi-agent systems with uncertain lur'e-type nonlinear dynamics," IET Control Theory \& Applications, vol. 7, no. 9, pp. 1249-1260, 2013.

[6] H. W. Zhang and F. L. Lewis, "Adaptive cooperative tracking control of higher-order nonlinear systems with unknown dynamics," Automatica, vol. 48, no. 7, pp. 1432-1439, 2012.

[7] Z. Peng, D. Wang, H. Zhang, and G. Sun, "Distributed neural network control for adaptive synchronization of uncertain dynamical multiagent systems," IEEE Transactions on Neural Networks and Learning Systems, vol. 25, no. 8, pp. 1508-1519, 2014.

[8] Y. Shi, Y. Yin, S. Wang, Y. Liu, and F. Liu, "Distributed leaderfollowing consensus of nonlinear multi-agent systems with nonlinear input dynamics," Neurocomputing, vol. 286, pp. 193197, 2018.

[9] C. Chen, C.-E. Ren, and T. Du, "Fuzzy observed-based adaptive consensus tracking control for second-order multi-agent systems with heterogeneous nonlinear dynamics," IEEE Transactions on Fuzzy Systems, vol. 24, no. 4, pp. 906-915, 2016.

[10] T. Yang, Impulsive Control Theory, vol. 272, Springer, Berlin, Germany, 2001.

[11] W. He, G. Chen, Q.-L. Han, and F. Qian, "Network-based leader-following consensus of nonlinear multi-agent systems via distributed impulsive control," Information Sciences, vol. 380, pp. 145-158, 2017.

[12] Y. Qian, X. Wu, J. Lü, and J.-A. Lu, "Second-order consensus of multi-agent systems with nonlinear dynamics via impulsive control," Neurocomputing, vol. 125, pp. 142-147, 2014.

[13] G.-S. Han, Z.-H. Guan, J. Li, D.-X. He, and D.-F. Zheng, "Multitracking of second-order multi-agent systems using impulsive control," Nonlinear Dynamics, vol. 84, no. 3, pp. 1771-1781, 2016.

[14] M. Akhmet, Principles of Discontinuous Dynamical Systems, Springer, New York, NY, USA, 2010.

[15] M. U. Akhmet, "On the general problem of stability for impulsive differential equations," Journal of Mathematical Analysis and Applications, vol. 288, no. 1, pp. 182-196, 2003.

[16] C. Li, Y. Zhou, H. Wang, and T. Huang, "Stability of nonlinear systems with variable-time impulses: B-equivalence method," International Journal of Control, Automation, and Systems, vol. 15, no. 5, pp. 2072-2079, 2017.

[17] X. Zhang, C. Li, and T. Huang, "Hybrid impulsive and switching Hopfield neural networks with state-dependent impulses," Neural Networks, vol. 93, pp. 176-184, 2017.

[18] H. Li, C. Li, and T. Huang, "Periodicity and stability for variabletime impulsive neural networks," Neural Networks, vol. 94, pp. 24-33, 2017.

[19] X.-J. Yang, C.-D. Li, and Q.-K. Song, "Muttag-Leffler stability analysis on variable-time impulsive factional-order neural networks," Neurocomputing, vol. 207, pp. 276-286, 2016.

[20] Q. Song, X. Yang, C. Li, T. Huang, and X. Chen, "Stability analysis of nonlinear fractional-order systems with variabletime impulses," Journal of The Franklin Institute, vol. 354, no. 7, pp. 2959-2978, 2017.
[21] S. Yang, C. Li, and T. Huang, "Synchronization of coupled memristive chaotic circuits via state-dependent impulsive control," Nonlinear Dynamics, vol. 88, no. 1, pp. 115-129, 2017.

[22] L. O. Chua, L. Pivka, and C. W. Wu, "A universal circuit for studying chaotic phenomena," Philosophical Transactions of the Royal Society A: Mathematical, Physical and Engineering Sciences, vol. 353, no. 1701, pp. 65-84, 1995. 


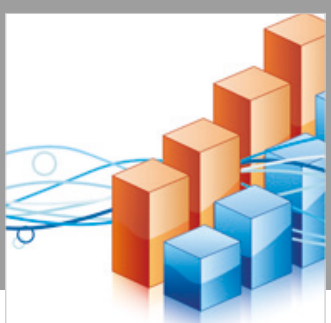

Advances in

Operations Research

\section{-n-m}
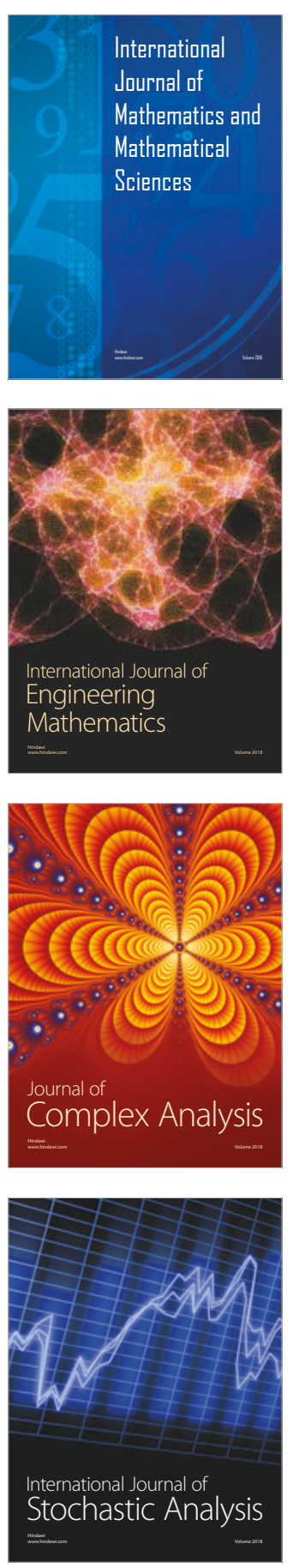
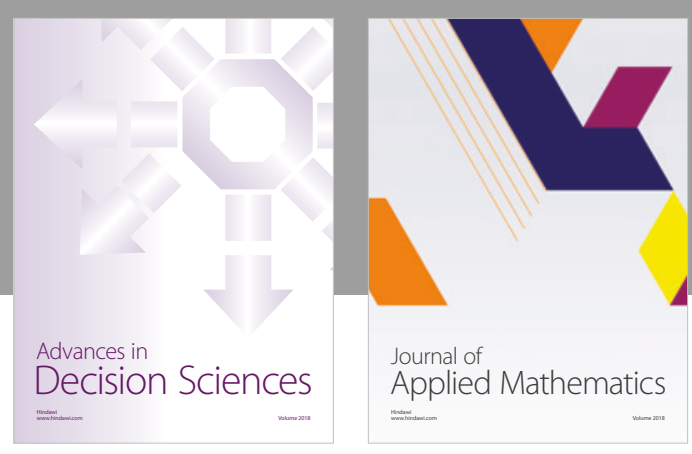

Journal of

Applied Mathematics
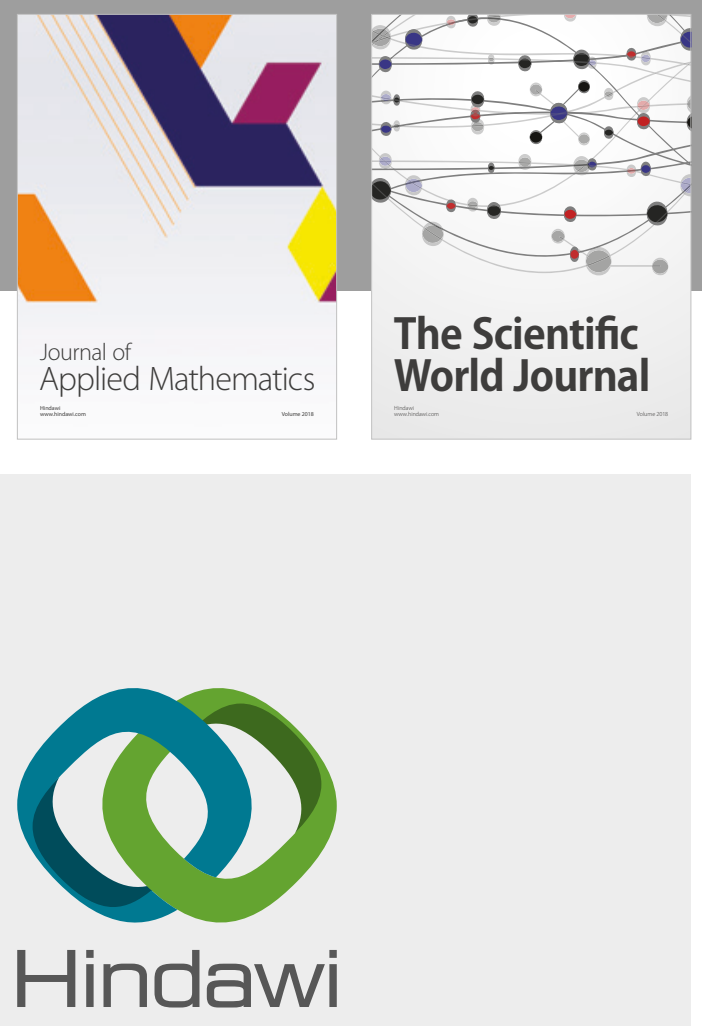

Submit your manuscripts at

www.hindawi.com

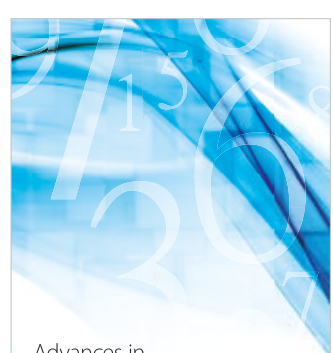

Advances in
Numerical Analysis
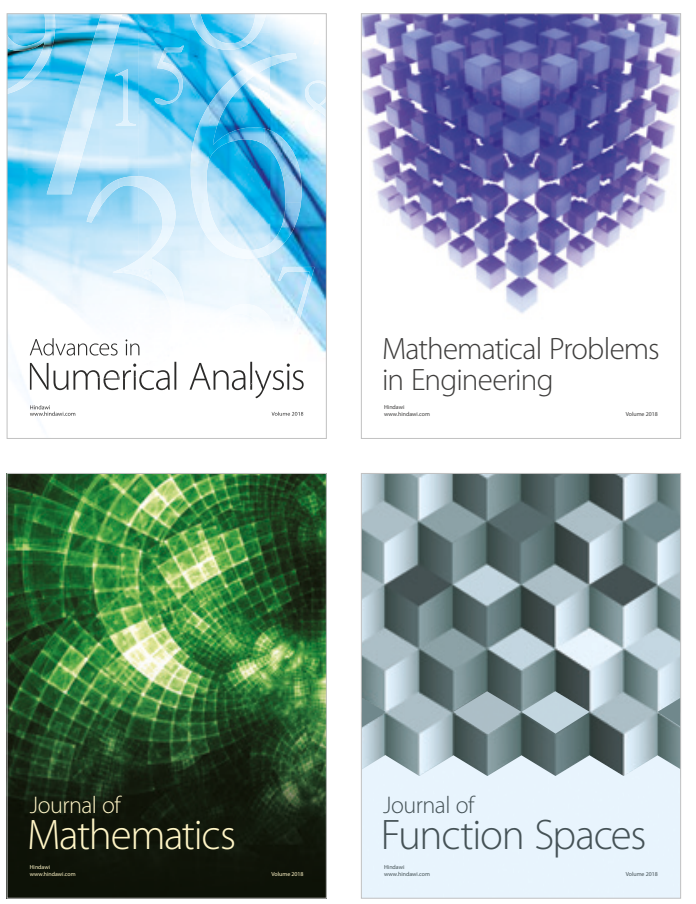

Mathematical Problems in Engineering

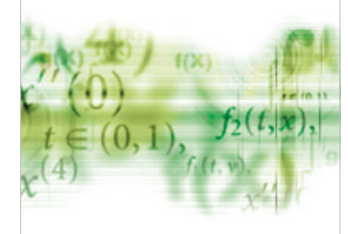

International Journal of

Differential Equations

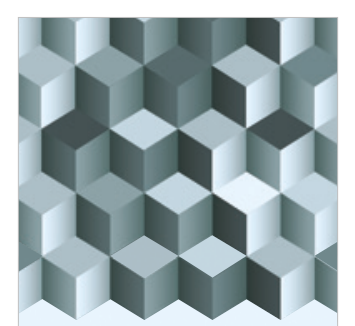

Journal of

Function Spaces
The Scientific

World Journal

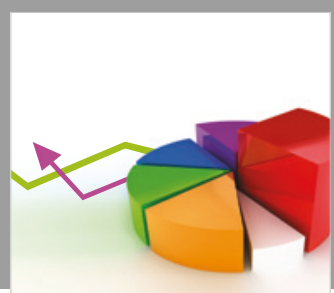

Journal of

Probability and Statistics
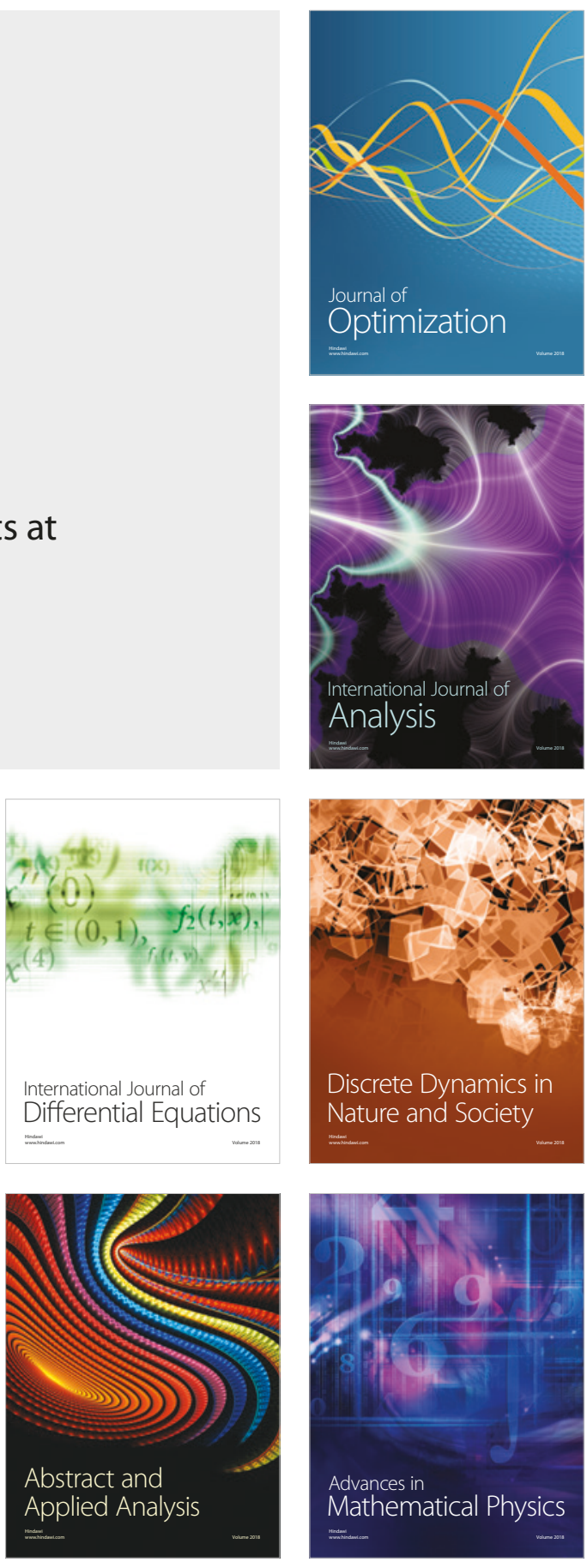